\title{
LA SCELTA PER LA REPUBBLICA
}

di Cesare Pinelli

\section{Una vittoria con forti fratture}

Sebbene la Costituzione provvisoria, in quanto accompagnata dalla prevista elezione di "una Assemblea Costituente [...] dopo la liberazione del territorio nazionale" (decreto legge luogotenenziale 25 giugno 1944, n. 151), già segnasse una prima rottura con l'ordinamento statutario, a renderla irreversibile fu la scelta degli elettori per la Repubblica in occasione del referendum del 2 giugno 1946. L'opzione opposta avrebbe significato una riconciliazione popolare con quell'ordinamento, ipotecando i lavori della Costituente ben al di là della disciplina della forma di governo.

Una minima disaggregazione dei dati elettorali basta peraltro a mostrare la estrema problematicità della vittoria della Repubblica. Intanto per uno scarto di due milioni di voti (poco più di 12.700 .000 a fronte dei poco più di 10.700 .000 voti per la Monarchia), con un milione e mezzo di schede bianche o nulle il cui mancato computo da parte della Corte di Cassazione provocò controversie ${ }^{1}$. Poi perché, a fronte di quello scarto, nella contestuale elezione dei membri della Costituente, i partiti antifascisti ottenevano una maggioranza schiacciante di seggi (circa 500 su 556). E soprattutto per la prevalenza della Monarchia nel Mezzogiorno come nel Lazio e in Abruzzo, che

1 Vista l'univoca interpretazione fino ad allora invalsa del termine "votanti" adoperato dal decreto legislativo luogotenenziale 16 marzo 1946, n. 98, tale da individuare "tutti coloro che abbiano esercitato il diritto di voto deponendo materialmente una scheda nell'urna", anziché "solo coloro che avevano compiuto una positiva manifestazione di volontà". Cfr. al riguardo la nota critica di C. EsPosito, La maggioranza nel referendum, a Cass., ord. 18 giugno 1946, in Giur.it., I, 1, c. 385 sgg.. 
malgrado la fine della guerra e la liberazione riproduceva un'Italia "tagliata in due"2.

In un'epoca lontana dalle passioni partitiche che animarono la prima fase della Repubblica, questi pur notissimi dati possono leggersi in una prospettiva diversa. Inducono a risalire alla decisione di affidare la scelta istituzionale al referendum (art. 1 decreto legislativo luogotenenziale 16 marzo 1946, n. 98), per ricercare i diversi percorsi che infine portarono tutti i suoi protagonisti a considerarla una strettoia indispensabile, a costo di interrogare un elettorato tanto lacerato. E, pur in veste mutata, il motivo del rapporto fra partiti e popolo si ripresenta nel dibattito alla Costituente sulla sottrazione a revisione costituzionale della "forma repubblicana".

\section{Un dilemma per molti italiani}

La decisione di affidare al referendum la scelta fra Monarchia o Repubblica è stata di solito ricostruita in studi dedicati a singoli suoi protagonisti o al periodo transitorio, col risultato di non inquadrare a sufficienza i dilemmi che investivano l'elettorato, con cui pure tutti i partiti si confrontarono pur implicando diversi calcoli contingenti per ciascuno.

La decisione è stata vista, così, come risultato di uno scambio che i partiti di sinistra avrebbero accettato dopo aver ottenuto la rinuncia della DC e delle destre alla previsione del voto obbligatorio ${ }^{3}$, o come l'unico modo per evitare tensioni ulteriori fino al pericolo di una guerra civile, appena scoppiata in Grecia ${ }^{4}$, o come esito di un solitario cedimento di Nenni alla soluzione referendaria sostenuta da De Gasperi ${ }^{5}$.

Le spiegazioni monocausali appaiono tuttavia poco convincenti, tanto più se si considera che, in quelle stesse sedute del 26 e 27 feb-

2 B. Croce, Quando l'Italia era tagliata in due. Estratto di un diario (luglio 1943giugno 1944), Bari, Laterza, 1948.

3 F. Catalano, La "nuova" democrazia italiana dopo il 1945, in S.J. Woolf (a cura), "Italia 1943-50", Bari, Laterza, 1974, p. 123, ripreso da S. LANARO, Storia dell'Italia repubblicana. Dalla fine della guerra agli anni novanta, Venezia, Marsilio, 1992, p. 45.

4 A. GAmbino, Storia del dopoguerra dalla liberazione al potere DC, Bari, Laterza, 1975, p. 139.

5 P. Scoppola, La proposta politica di De Gasperi, Bologna, il Mulino, 1977, p. 229. 
braio, il Consiglio dei ministri dovette risolvere altre due questioni non meno divisive, quale il tenere il referendum contestualmente alle elezioni o nel corso dei lavori dell'Assemblea, e l'attribuire il potere legislativo alla Costituente, secondo quanto poteva inferirsi dal decreto legge luogotenenziale n. 151 del 1944, o mantenerlo al Governo nella forma del decreto legislativo luogotenenziale.

Per giunta, la decisione fu lo sbocco di un percorso durato quasi due anni. Nella seduta del Consiglio dei ministri del 22 giugno 1944 sul testo approvato con decreto legge luogotenenziale n. 151, i fautori del referendum avevano già cercato di far passare la loro tesi, col risultato di lasciare margini di incertezza sulla formulazione adottata, secondo cui "Dopo la liberazione del territorio nazionale, le forme istituzionali saranno scelte dal popolo italiano che a tal fine eleggerà, a suffragio universale diretto e segreto, una Assemblea Costituente per deliberare la nuova costituzione dello Stato. I modi e le procedure saranno stabiliti con successivo provvedimento" (art. 1). Restava dubbio se le parole "a tal fine" dovessero riferirsi anche alle "forme istituzionali", o se la scelta di tali forme fosse viceversa da riferirsi al popolo italiano indipendentemente dall'elezione della Costituente ${ }^{6}$.

Certo è che, appena due giorni dopo, emerge la principale contrapposizione che dominerà il dibattito politico. Nenni avverte che "Una delle insidie di chi si riveste dell'armatura della libertà per meglio combatterla, è quella del plebiscito, affiorata già in questi giorni a Salerno [...] Sotto la maschera plebiscitaria abbiamo già visto affiorare il volto della reazione sociale. Noi socialisti non siamo contrari, in linea di principio, alle consultazioni del referendum, ma il problema, anzi i problemi da porre davanti alla Costituente, non si risolvono con un semplice 'sì' o con un semplice "no" "7. Gonella gli replica che il referendum "riporta direttamente il popolo sulla scena dello Stato", poiché con questo strumento "lo si rende capace di essere non più oggetto ma soggetto di diritti politici, non più amorfa massa passiva ma cosciente ed attivo artefice di una politica basata sul consenso"». Nenni, tanto più

6 Cfr. A. Pichierri, La Costituzione provvisoria. L'ordinamento dello Stato tra Fascismo e Repubblica, Taranto, Mandese, 1996, p. 243.

7 P. NenNi, La costituente sancita dalla legge. Una vittoria della democrazia, in “Avanti!", 24 giugno 1944.

8 G. Gonella, Autogoverno e referendum, in "Il Popolo", 26 giugno 1944. 
dopo aver registrato il silenzio di De Gasperi sulla scelta della DC in ordine alla forma istituzionale, ribatte allora che "Il referendum è una forma diretta di democrazia che ha tutta la nostra simpatia. Ma nell'Italia che esce da ventidue anni di fascismo, il referendum è una cabala reazionaria"9.

Nella polemica era nel frattempo intervenuto Crisafulli, per dire che mentre nel referendum "il popolo viene direttamente interpellato in merito a determinati provvedimenti legislativi che si ritengono di speciale importanza politica", nel plebiscito "il popolo viene interrogato non già intorno ad atti che, sia pure eventualmente modificandolo in qualche parte, rientrano nel quadro e nel funzionamento di un dato ordine costituzionale precedentemente stabilito, ma addirittura proprio nella validità iniziale di un tale ordine costituzionale"10. Dove l'opportuna distinzione fra i due istituti mirava a supportare l'originario rifiuto dei comunisti per l'opzione referendaria.

La scelta del febbraio ' 46 ebbe dunque una lunghissima incubazione, in un confronto ricco di argomenti da una parte e dall'altra. Al centro del dibattito non furono però l'interpretazione del decreto legge luogotenenziale del '44, o la distinzione giuridica fra referendum e plebiscito, o l'associazione dello strumento referendario a forme di ricerca del consenso tipiche dei totalitarismi. Il fatto è che un autentico dilemma sul significato delle opzioni istituzionali in campo attraversava l'elettorato, e conseguentemente i partiti.

Il discredito nei confronti di Vittorio Emanuele III era generalizzato, nonostante che per la gente comune l'istituzione monarchica e con essa la dinastia dei Savoia continuassero a rappresentare l'unità nazionale e riflettessero il bisogno di "una certezza istituzionale definita e non soggetta ad altri colpi se non a quelli voluti dal destino naturale" 11 . All'opposto, i partiti fautori della Repubblica godevano di un largo credito popolare. Ma la sostenevano come "una scelta della ragione" 12 che non bastava a definirne i contorni, dopo che la componente repubblicana della Resistenza, di gran lunga prevalente, aveva acco-

9 P. Nenni, Risposta a De Gasperi, in “Avanti!”, 26 luglio 1944.

10 V. CRISAFUlLi, Referendum e Costituente, in "L' Unità", 1 luglio 1944.

${ }^{11}$ E. Di Nolfo, Le paure e le speranze degli italiani (1943-1953), Milano, Mondadori, 1986, p. 147.

${ }^{12}$ E. Di Nolfo, Le paure e le speranze degli italiani, cit., p. 148. 
munato partiti che ambivano "a tipi di repubbliche diverse, da quella dei soviet a quella democratico-borghese"13. In quelle condizioni, associare la Repubblica a un "salto nel buio" non era solo frutto della propaganda avversaria.

Del dilemma si possono indicare vari sintomi. A partire dal tentativo intrapreso da Croce prima della svolta di Salerno di indurre il re ad abdicare, non a favore del figlio Umberto, che in parte ne aveva condiviso le colpe, ma del primogenito di lui, l'ancora minorenne principe di Napoli, con conseguente creazione di una reggenza: la convinzione che Vittorio Emanuele III avesse tradito l'Italia consegnandola a Mussolini non scalfiva i sentimenti monarchici del filosofo napoletano ${ }^{14}$.

Non fu nemmeno un caso se, più tardi, il Luogotenente accompagnò la sanzione e la promulgazione del decreto legislativo luogotenenziale n. 98 del 1946 con una lettera in cui accennava al proclama, fino ad allora poco diffuso, lanciato dal padre agli italiani al momento della sua nomina a Luogotenente (12 aprile 1944) e in cui si richiamava ai plebisciti sulle annessioni come un "patto tra popolo e monarchia" che, se confermato, avrebbe costituito "il fondamento di una Monarchia rinnovata" e destinata ad attuare pienamente "l'autogoverno popolare e la giustizia sociale"15.

Ancora, l'abdicazione di Vittorio Emanuele in favore del figlio, sopraggiunta in piena campagna elettorale (9 maggio 1946), non suscitò soltanto l'ira di Togliatti e un decisivo richiamo di De Gasperi alla perdurante operatività della tregua istituzionale del giugno 1944 onde escludere l'ipotesi di un re con poteri transitori di fronte a un'assemblea tendenzialmente repubblicana ${ }^{16}$. Perché "il gesto dell'abdicazione colpì la fantasia del popolino e rianimò molti monarchici quasi scoraggiati. La personalità affabile di Umberto, che si rivelava nelle fotografie come quel vero padre di famiglia, che aveva tanto stentato a diventare

13 U. Terracini, Come nacque la Costituzione. Storia inedita dell'Assemblea Costituente, Intervista di P. Balsamo, Roma, Editori Riuniti, 1978, p. 15.

${ }^{14}$ G. SASso, Prefazione a "Dall'Italia tagliata in due all'Assemblea costituente. Documenti e testimonianze dai carteggi di Benedetto Croce", Bologna, il Mulino, 1998, p. 14 sgg..

${ }^{15}$ Cfr. G. Romita, Dalla Monarchia alla Repubblica, Milano, Mursia, 1966, p. 101.

16 P. Craveri, De Gasperi, Bologna, il Mulino, 2006, p. 235; G. Andreotti, Gli “anni brevi" della Costituente, in "Studi per il ventesimo anniversario dell'Assemblea Costituente”, Firenze, Vallecchi, 1969, vol. I, p. 465. 
nella vita, guadagnò rapidamente consensi su consensi. Il sentimentalismo famigliare così profondamente radicato nel popolo italiano si rovesciò all'improvviso sulla carta monarchica" 17 .

Emblematica appare altresì una conversazione di Nenni con l'allora Ministro degli Interni Romita, da questi riportata: "La monarchia ha troppi torti - gli feci osservare io - perché il popolo non ne faccia giustizia. Sì, d'accordo - egli diceva, più o meno - ma settantacinque anni di regime monarchico, con una propaganda capillare, quotidiana, che ha accompagnato ogni singolo cittadino dalla scuola sino all'età adulta, e la presenza al Quirinale di Umberto, dipinto come antifascista e simbolo dell'unità nazionale, potrebbero darci amare sorprese"18.

\section{La decisione di affidare la scelta al referendum:} i percorsi dei dirigenti politici

Se la decisione matura per tutti sullo sfondo di un dilemma "fra sentimento e ragione" 19 , diverse erano le visioni che ne avevano i protagonisti, condizionate dai ricordi del fallimento della democrazia nel primo dopoguerra.

In un passaggio del discorso di De Gasperi al I Congresso della DC (24 aprile 1946), dove parla del referendum come di "un metodo pacificatore [...] perché dopo questa decisione arbitrale del popolo non ci potranno essere contestazioni di sorta", si è vista l'ottica di "chi, avendo visto fallire la stabilizzazione seguita al primo dopoguerra, era assai preoccupato della risorgenza di qualcosa di simile"20. Né il giudizio era imposto dalla contingenza elettorale, dato che risale al 12 novembre 1944 una lettera a Sturzo in cui affermava: "Per me il referendum ha un grande valore morale, perché dà il senso democratico e pacificatore di una suprema decisione popolare e di un consenso esplicito della maggioranza alla nuova forma dello stato" 21 .

${ }^{17}$ L. Valiani, L'Italia di De Gasperi (1945-1954), Firenze, Le Monnier, 1982, p. 56.

18 G. Romita, Dalla Monarchia alla Repubblica, cit., p. 77.

${ }^{19}$ G. Romita, Dalla Monarchia alla Repubblica, cit., p. 77, il quale era favorevole alla soluzione referendaria ben prima di Nenni.

20 P. PombenI, La questione costituzionale in Italia, Bologna, il Mulino, 2016, p. 154, anche per la citazione del passaggio del discorso degasperiano.

${ }^{21}$ P. POMBENI, La questione costituzionale in Italia, cit., p. 89. 
In Nenni giocava invece l'intento di riuscire là dove aveva fallito nel biennio 1919-1921, quando era già "convinto che la Repubblica e la Costituente - e non già i soviet e la dittatura del proletariato - fossero gli obiettivi rivoluzionari possibili, e che la classe che doveva farli suoi era la classe operaia, e il partito che poteva realizzarli era il Partito socialista"22.

L'originario giudizio sul referendum come "cabala reazionaria" all'indomani del fascismo si inscrive in questo solco, oltre a riflettere le suggestioni giacobine di tutta la sinistra che torneranno ad avvertirsi alla Costituente. Nondimeno i comunisti si opposero alla soluzione referendaria più cautamente di Nenni e dei socialisti: giocava in loro l'estremo pragmatismo nei confronti della monarchia inaugurato dalla svolta di Salerno, in cui del resto già i contemporanei videro il capolavoro politico di Togliatti.

La Repubblica e la Costituente continueranno a lungo ad apparire a Nenni un tutto inscindibile. Al di là delle pressioni di De Gasperi, il quale chiese agli Alleati, come aveva fatto Bonomi, di convincerlo a cambiare posizione ${ }^{23}$, la sua conversione al referendum sarà frutto di un apprendimento che matura nel corso del mandato di Ministro per la Costituente del Governo Parri e del I Governo De Gasperi. Salutata la nomina come un riconoscimento al "dirigente del partito che nei termini più risoluti ha posto il problema della Costituente come problema di oggi e che di fronte alla Costituente si era impegnato ad assumere una posizione nettamente, risolutamente, intransigentemente repubblicana" 24 , nei mesi successivi si rende conto che 1'Italia non può essere conquistata alla causa della Costituente e della Repubblica in una pro spettiva giacobina. Lo sforzo suo e del Capo di gabinetto Massimo Severo Giannini consisterà anzi nel dimostrare che la Costituente può essere il contrario di una Convenzione, che la borghesia associa alla ghigliottina ${ }^{25}$. D'altro canto, restava in lui il timore che gli elettori potessero confondere il referendum coi plebisciti

22 P. NenNI, Intervista sul socialismo italiano, a cura di G. Tamburrano, Bari, Laterza, 1977, p. 28.

${ }^{23}$ A. GAmBIno, Storia del dopoguerra, cit., p. 135 sgg..

24 P. NenNi, Con il popolo e per il popolo, in “Avanti!”, 19 giugno 1945.

25 M.S. Giannini, Nenni al Ministero per la Costituente, in "Nenni dieci anni dopo", a cura della Fondazione Nenni, Roma, Lucarini, 1990, p. 54. 
dell'Unificazione. Solo alla vigilia del Consiglio dei ministri del 26 febbraio 1946 annota nel diario che "Se prolunghiamo la polemica sui poteri della Costituente e sul referendum si avranno crisi di governo e salto nel buio", e poi aggiunge: "Il referendum istituzionale è per la Corte un sostitutivo dei plebisciti ma può anche diventare un'altra cosa se contestuale alle elezioni per la Costituente" 26.

Nonostante il giudizio di Scoppola sulla sua strumentalità politica in quel frangente ${ }^{27}$, dire che il referendum "può anche diventare un'altra cosa" equivale a correre un rischio, e per raggiungere un obiettivo non di parte ma generale. Né lo spostamento di qualche mese del referendum proposto da De Gasperi rifletteva una visione più lungimirante della contestualità fra referendum ed elezioni. Sarà questa soluzione a prevalere, e a far superare un contrasto che sembrava difficile da risolvere ${ }^{28}$.

Secondo Andreotti, nella seduta del 27 febbraio, "iniziata alle nove del mattino e terminata - con un intervallo di venticinque minuti per la colazione - alle undici della sera", la decisione di scindere il voto in due operazioni distinte fu "particolarmente travagliata", e fu "il sostegno pacato e aperto di De Gasperi" a prevalere "sulla tesi inizialmente sostenuta dalla maggioranza dei ministri" 29 . Dal verbale risulta peraltro che il Consiglio di Gabinetto aveva già sciolto il nodo, e che la discussione si animò soprattutto per l'opposizione dell'azionista Cianca alla soluzione referendaria ${ }^{30}$. In ogni caso, ricorda Romita, "Trattammo le diverse questioni con spirito appassionato, cercando ciascuno di far prevalere le proprie tesi, ma non irrigidendoci mai su posizioni preconcette"31.

\section{L'arte della differenziazione per comporre conflitti}

Fu allora che, per comporre conflitti, i partiti cominciarono a praticare l'arte della differenziazione. Fra i tanti sintomi ulteriori, si ricorda

26 A. GAmBino, Storia del dopoguerra, cit., p. 138.

27 P. SCOPPOLA, La proposta politica di De Gasperi, cit., p. 225.

28 P. Craveri, De Gasperi, cit., p. 216.

${ }^{29}$ G. Andreotti, Gli “anni brevi” della Costituente, cit., p. 464.

30 Verbale del Consiglio dei ministri del 27 febbraio 1946: si veda A. PiCHIERRI, La Costituzione provvisoria. L'ordinamento dello Stato tra Fascismo e Repubblica, cit., p. 380 sgg..

31 G. Romita, Dalla Monarchia alla Repubblica, cit., p. 99. 
la segnalazione, il 28 giugno, di una rosa di candidati monarchici e meridionali alla carica di Capo provvisorio dello Stato, da cui, dopo la rinuncia di Benedetto Croce e il veto socialista a Vittorio Emanuele Orlando, scaturì il nome di Enrico De Nicola ${ }^{32}$. Lo attesta pure, nel luglio successivo, il rigetto della proposta Calamandrei di assegnare all'Assemblea tutti i poteri di un parlamento, sul presupposto che l'art. 3 del decreto legislativo luogotenenziale n. 98 del 1946 fosse stato superato dalla nuova legittimazione acquisita dalla Costituente ${ }^{33}$. Infine, nella stessa prospettiva può leggersi la doppia limitazione che avrebbe molto più tardi incontrato la conventio ad excludendum nei confronti del PCI, circoscritta al livello nazionale ed esclusa per le leggi di attuazione costituzionale e i regolamenti parlamentari.

Vanno però considerati i contesti. Nella prassi repubblicana avviata a stento negli anni Cinquanta e consolidata nel successivo trentennio, l'arte della differenziazione diventerà sempre più un esercizio autoreferenziale, utile a sostenere posizioni di rendita da una parte e dall'altra. All'epoca dell' instaurazione della Repubblica, comportava invece i rischi del politico-imprenditore descritti da Max Weber. Soprattutto nel caso del referendum, dove veniva interrogato un elettorato che tutti sapevano assai diviso indipendentemente dall'esito, e che tale si mostrò il 2 giugno 1946. A quel punto, occorreva fargli accettare nell'immediato la regola della maggioranza: solo più tardi, e a costo di reciproche rinunce, si sarebbe potuto contare sulle virtù trasformative innescate dall'approvazione del testo costituzionale.

L'operazione si rivelò difficile fin dalla sanguinosa rivolta di Napoli e dalle forti tensioni in Puglia e in Sicilia fra il 7 e il 12 giugno $^{34}$. La fine della monarchia sconvolgeva antichi equilibri, con la definitiva scomparsa dei valori di stabilità e di continuità cui era associata: "Lo sguardo 'verticale' rivolto all'autorità paterna del sovrano nell'inno nazionale monarchico Cunservet Deus su Rei si trasforma nell'orizzontale ed egualitario Fratelli d'Italia repubblicano"35.

32 A. Lepre, Storia della prima Repubblica. L'Italia dal 1943 al 2003, Bologna, il Mulino, 2004, p. 75.

${ }^{33}$ U. TERracini, Come nacque la Costituzione, cit., p. 62.

${ }^{34}$ G. Romita, Dalla Monarchia alla Repubblica, cit., p. 219 sgg..

${ }^{35}$ R. BodEI, Il noi diviso. Ethos e idee dell'Italia repubblicana, Torino, Einaudi, 1998, p. 35 . 


\section{Il dibattito dei Costituenti sulla sottrazione della "forma repubblicana" a revisione costituzionale}

La questione delle perduranti fratture che dividono gli italiani riaffiora dai lavori della Costituente sulla sottrazione a revisione costituzionale della forma repubblicana.

Nella seduta di apertura dei lavori dell'Assemblea, il Presidente Orlando osservava che la piena libertà di scelta della Costituente "ha però un limite che fu fissato direttamente dalla stessa volontà popolare, con un atto che può qualificarsi di democrazia diretta. E questo limite consiste in ciò: la forma di governo, per quanto riguarda la qualità del capo dello Stato, è la Repubblica. L'istituto che vi corrisponde è dunque diventato il simbolo dello Stato; è attraverso di esso che la Nazione d'Italia si personifica come organica unità indissolubile" 36 . Ben presto, comunque, si vide che la scelta per la Repubblica, per tutti fuori discussione quale limite al potere dell'Assemblea, diventava oggetto di disputa in quanto la si considerasse limite irrevocabile per il potere costituito. La questione si pose prima ancora di stabilire se la Costituzione dovesse essere rigida o flessibile. Nelle sedute del 28 e 29 novembre 1946 della I Sottocommissione della Commissione dei Settantacinque, Togliatti propose un emendamento aggiuntivo all'art. 1 così formulato: "La forma repubblicana dello Stato non può essere messa in discussione né davanti al popolo, né davanti alle Assemblee Legislative".

Oltre al monarchico Lucifero, che rivendica il diritto del popolo di poter in futuro cambiare quella forma, e al demolaburista Cevolotto, che ritiene inutile la formulazione di fronte a un diverso orientamento della maggioranza degli elettori, interviene il Presidente Tupini per chiedere a Togliatti se in base alla sua proposta debba considerarsi reato un articolo di giornale che discuta la forma repubblicana. Dopo averlo negato, trattandosi di formula da inserire in Costituzione anziché nel codice penale, Togliatti presenta tuttavia un emendamento alternativo, secondo cui "La forma repubblicana dello Stato non può essere oggetto di proposta di revisione costituzionale".

Moro chiarisce a quel punto che "il problema in esame è soprattutto politico, perché ci si trova di fronte ad una istituzione repubblicana di

\footnotetext{
${ }^{36}$ Atti dell'Assemblea Costituente, seduta del 25 giugno 1946, p. 2.
} 
recente creata in Italia, mentre ancora sussistono forze politiche disorganizzate, le quali alimentano le speranze di alcuni strati del popolo in un ritorno monarchico, sfruttandone le ingenuità [...] La Sottocommissione deve preoccuparsi di questa situazione, e affermare una norma che dica al popolo italiano che vi è una sanzione sovrana che non può essere messa in discussione. Tale norma può trovare la sua più opportuna espressione in una formula che non si preoccupi troppo della revisione della Costituzione, ma delle speranze che vengono alimentate nell'ingenuità popolare, e che costituiscono un pericolo per la stabilità dell'ordine costituito in Italia" 37.

Il giorno dopo, l'adesione di Mancini alla posizione di Moro, "perché la democrazia, che si identifica con la Repubblica, in tanto esiste in quanto sappia difendere sé stessa", conferma che i tre maggiori partiti si schieravano a favore della proposta Togliatti. Ma subentrano obiezioni di carattere tecnico, giacché per Basso, De Vita e Caristia essa equivarrebbe a prevedere una procedura di secondo grado per la revisione costituzionale della forma di Stato, sulla scia, dicono, dell'esempio francese della IV Repubblica. E Cevolotto si inserisce nella discussione per proporre una remissione della questione alla Seconda Sottocommissione, dedicata all'ordinamento costituzionale della Repubblica. Moro propone allora una formulazione per cui "L'adozione della forma repubblicana dello Stato è definitiva e non può essere oggetto di normale procedimento di revisione della Costituzione", che incontra il favore di Togliatti e Mancini e viene approvata con quindici voti favorevoli e due contrari38.

Nella seduta dell'Aula del 3 dicembre 1947, approvata la formula poi contenuta nell'art. 138, cui si decise di far seguire la clausola di sottrazione a revisione della forma repubblicana, la discussione si incentrò invece sugli emendamenti Benvenuti e Laconi, volti a prevedere la sottrazione a revisione, rispettivamente, dei diritti di libertà e del diritto al lavoro ${ }^{39}$.

${ }^{37}$ La Costituzione della Repubblica Italiana, a cura del Segretariato Generale della Camera dei Deputati, VI, Roma, 1971, p. 433 sgg..

${ }^{38}$ La Costituzione della Repubblica Italiana, cit., p. 439 sgg..

39 Atti dell'Assemblea Costituente, p. 2764 sgg.. Sul significato di questi emendamenti e del loro rigetto si può vedere C. PInELLI, Costituzione rigida e Costituzione flessibile nel pensiero dei Costituenti italiani, Milano, Giuffrè, 1981, ed. provv., p. 92 sgg.. 
Il dibattito in Sottocommissione interessa per la compresenza di due motivi all'epoca fra loro intrecciati. La sottrazione a revisione disposta per la scelta repubblicana sarebbe servita sia a scoraggiare attentati alla nuova legalità ad opera di "forze politiche disorganizzate", ma anche in grado di agire efficacemente sull'immaginario popolare, sia a sancire un limite alla disponibilità del potere di normazione costituzionale tale da imporsi a qualsiasi maggioranza parlamentare.

Le obiezioni di inutilità non avevano pregio. In primo luogo perché alla dichiarazione di irreversibilità della forma repubblicana non poteva disconoscersi un alto valore simbolico, pur in assenza dei congegni sanzionatori di una "democrazia che si difende" di lì a breve messi a punto nella Grundgesetz. In secondo luogo quelle obiezioni provavano troppo, dovendosi estendere alla scelta per la rigidità costituzionale, del pari suscettibile di venire travolta di fatto da una maggioranza di dissenzienti irriducibili.

Proprio perché tutt'altro che inutile, la formula si prestava piuttosto al rilievo, implicitamente ricavabile dai dissensi dei monarchici e dei qualunquisti, di forzare la volontà di un elettorato espressosi nei termini ricordati all'inizio, fino a escludere che una frattura sul punto potesse in futuro riprodursi alla stregua del diritto costituzionale vigente.

Non a caso, la divergenza di vedute circa il carattere solo politico $\mathrm{o}$ anche giuridico dell'immodificabilità della forma repubblicana continuerà ad avvertirsi all'indomani dell'approvazione della Costituzione.

Al Presidente del Consiglio De Gasperi, che in linea con la maggioranza dei Costituenti si era espresso nel primo senso, Elia replicò che "Per impostare con esattezza il problema bisogna rifarsi a concezioni giuridiche, le quali, pur non uscendo dal campo del diritto positivo, sappiano guardare oltre le norme scritte, e, con più viva consapevolezza della realtà storica, si propongano di identificare in una zona più profonda e più remota, i caratteri fondamentali che distinguono tutto un ordinamento; è necessario ricorrere cioè alle recenti ricerche sui concetti di Costituzione e di regime politico"40. E così sviluppava le ricerche di Mortati e di Gueli: "non si esclude naturalmente che possa esser mutata per via pacifica o anche apparentemente

${ }^{40}$ L. Elia, Possibilità di un mutamento istituzionale in Italia, in "Costituzione, partiti, istituzioni”, Bologna, il Mulino, 2009, p. 12. 
legale (mediante gli organi e le procedure stabilite dalla Costituzione) la forma repubblicana di governo; si esclude soltanto che si resti nel quadro della vigente Costituzione, che l'atto di revisione possa imputarsi alla fonte giuridica degli atti precedenti, che resti immutato il tipo di Stato: ci troveremmo sempre dinanzi ad una instaurazione di fatto"41.

Il verso della tessitura fra 'politico' e 'giuridico' non poteva essere più esplicito. Posta l'inscindibile connessione sistematica dell' art. 139 con l'art. $1^{42}$, la distinzione legalità/legittimità restituiva al dominio del giuridico una zona primaria dell'ordinamento oggetto di una recentissima contesa politica. Erano le prime righe di un'opera nuova. Anche i costituzionalisti avevano voltato pagina.

\begin{abstract}
At the referendum held on 2 June 1946, the Republic prevailed over the Monarchy. This marked a watershed in Italian history, although the electorate appeared strongly divided along territorial lines, given the majority gained by monarchists in the South and partly in the Center of the country. The author reconstructs the complex decision-making process that brought the new Italian political parties to give the electorate, rather than the contextually elected Con-
\end{abstract}

stituent Assembly, the opportunity of deciding on the issue. In particular, the essay attempts to explain why such choice, formally enacted with the Lieutenant's legislative decree of 16 March 1946, n. 98, engendered the risk of a deep division of the country. And how, notwithstanding such risk, the protagonists of the new political system finally reached the awareness that the electors should be trusted with a view to prepare a common constitutional future.

${ }^{41}$ L. Elia, Possibilità di un mutamento istituzionale in Italia, cit., p. 12.

${ }^{42}$ L. Elia, Possibilità di un mutamento istituzionale in Italia, cit., p. 11. 